\title{
Esophagogastric Junction Carcinomas - Discriminating Histological Types Through Immunohistochemistry
}

\author{
KUNIO MOCHIZUKI, TETSUO KONDO, NAOKI OISHI, IPPEI TAHARA, \\ TOMOHIRO INOUE, KAZUNARI KASAI, TADAO NAKAZAWA and RYOHEI KATOH \\ Department of Pathology, School of Medicine, University of Yamanashi, Chuo, Japan
}

\begin{abstract}
Background/Aim: Although differentiating squamous cell carcinoma (SCC) from adenocarcinoma (AC) at the esophagogastric junction (EGJ) is important for the choice of treatment, this can occasionally be difficult with small biopsy specimens. Therefore, the purpose of this study was to determine the most useful immunomarker panel for discriminating between SCC and AC of the EGJ. Materials and Methods: We analyzed 15 SCCs and 26 ACs of the EGJ obtained surgically using immunohistochemistry. Results: The sensitivities of p40, p63 and cytokeratin 5/6 were 100\% with specificities of $88 \%, 46 \%$ and $81 \%$, respectively, for SCC. The sensitivities of CAM5.2, caudal-type homeobox 2 (CDX2), mucin-5AC (MUC-5AC) and MUC-6 were 100\%, $81 \%, 77 \%$ and $85 \%$ with specificities of $27 \%, 100 \%, 87 \%$ and $87 \%$ for AC. Conclusion: We demonstrated that a twomarker panel of p40 and CDX2 is highly sensitive and specific.
\end{abstract}

Squamous cell carcinoma (SCC) and adenocarcinoma (AC) are the predominant types of malignant tumors found in the human upper digestive tract: SCC in the esophagus and AC in the stomach. Consequently, when a poorly differentiated carcinoma from one of these organs has equivocal histology, clinical management is often based on the assumption that the tumor is either an SCC if found in the esophagus or an $\mathrm{AC}$ if found in the stomach. However, both SCCs and ACs commonly occur at the esophagogastric junction (EGJ). It is important therefore to determine the accurate histological subclassification of EGJ carcinomas (SCC or AC) in order to provide the best operative method, chemotherapy and

Correspondence to: Kunio Mochizuki, MD, Ph.D., Department of Pathology, School of Medicine, University of Yamanashi, 1110 Shimokato, Chuo, Yamanashi, 409-3898, Japan. Tel: +81 552739529, Fax: +81 552739534, e-mail: kuniom@yamanashi.ac.jp

Key Words: Squamous cell carcinoma, adenocarcinoma, esophagogastric junction, immunohistochemistry. radiotherapy for treatment. Furthermore, recent advances in targeted therapies have increased the need for accuracy in the classification of EGJ carcinomas. For example, human epidermal growth factor receptor 2 (HER2)-targeted therapy is currently recommended for treatment of advanced HER2positive ACs of the EGJ (1).

Precise identification of a carcinoma has become imperative, particularly in patients with advanced disease or metastasis, since the majority of these patients are not candidates for surgical resection of the carcinoma. In these patients, biopsy of the tumor is frequently performed to obtain tumor tissue for the diagnosis and histological evaluation of the tumor. Although histomorphological examination of hematoxylin and eosin (HE)-stained tissue slides can differentiate between SCC and $\mathrm{AC}$ of the EGJ, an accurate classification can be difficult in small biopsy specimens due to a low number of tumor cells, lack of characteristic architecture, artifacts in specimen preparation, and differentiation and heterogeneity of the carcinoma. Poorly differentiated carcinomas are particularly difficult to classify since they lack specific architectural or cytological features of either SCC or AC.

There are numerous immunohistochemical markers that are used to distinguish pulmonary SCC from AC (2). The p63 antibody that is routinely used in most pathology laboratories is 4A4. The p63/4A4 antibody recognizes both TAp63 and $\Delta \mathrm{Np} 63$ isoforms and is, therefore, a 'pan-p63' marker. Antibodies that differentiate between p63 isoforms, particularly the p40 antibody that recognizes only $\Delta \mathrm{Np} 63$ and not TAp63, have been available for several years (3-5). Recently, Bishop et al. reported that the sensitivity of p40 for pulmonary SCC was $100 \%$ and specificity was $98 \%$, while those of p63/4A 4 were $100 \%$ and $60 \%$, respectively (3). This shows that the p40 antibody is highly squamous cell-specific, unlike the p63/4A4 antibody. Furthermore, several studies have shown a high frequency (89-100\%) of p40 expression in pulmonary SCCs and a very low frequency (0-3\%) in pulmonary ACs (3-5).

Although there are reports demonstrating the differentiation of SCC from AC of the esophagus using 
Table I. Clinicopathological characteristics of patients with squamous cell carcinoma (SCC) or adenocarcinoma (AC) of the esophagogastric junction.

\begin{tabular}{lcc}
\hline Characteristics & $\begin{array}{c}\text { Patients with } \\
\text { SCC }(\mathrm{n}=15)\end{array}$ & $\begin{array}{c}\text { Patients with } \\
\text { AC }(\mathrm{n}=26)\end{array}$ \\
\hline Gender, $\mathrm{n}$ & & \\
Male & 11 & 23 \\
Female & 4 & 3 \\
Age & & \\
Mean (years) & 70 & 66 \\
Range & $57-85$ & $41-80$ \\
pT-Category*, n & & \\
pT1a & 3 & 3 \\
pT1b & 0 & 5 \\
pT2 & 0 & 2 \\
pT3 & 12 & 2 \\
pT4a & 0 & 0 \\
pT4b & 0 & 24 \\
\hline
\end{tabular}

*Evaluated according to the TNM classification of carcinoma of the esophagus.

immunohistochemical markers[p40, p63, cytokeratin 5/6, caudal-type homeobox 2 (CDX2), mucin-5AC (MUC-5AC), sex determining region Y-box 2 (SOX2) and anterior gradient 2 (AGR2)], the most useful marker panel has not yet been determined (6-8). ACs of the esophagus arise predominantly from columnar ('Barrett') mucosa in the lower third of the esophagus, and ACs that straddle the junction of the esophagus and stomach are designated as tumors of the EGJ (derived from esophagus or stomach). SCCs that occur at the EGJ are considered carcinomas of the distal esophagus, even if they cross the EGJ (9).

In this study, we investigated the utility of p40, p63 and cytokeratin 5/6 (as markers of squamous differentiation), anti-cytokeratin CAM5.2 (marker of glandular epithelial differentiation), CDX2 (marker of intestinal epithelial differentiation), MUC-5AC (marker of gastric foveolar epithelial differentiation) and MUC-6 (marker of cardiac gland, mucous neck cell or pyloric gland differentiation) and compared their sensitivities and specificities as markers for discriminating between SCC and AC of the EGJ.

\section{Materials and Methods}

Patients. We collected 15 samples of SCC and 26 samples of AC of the EGJ obtained surgically between January 2000 and December 2016 at the University of Yamanashi Hospital. We excluded resection specimens acquired after neoadjuvant chemotherapy or radiation therapy from this study group. All 15 SCCs crossed or reached the EGJ. According to the Siewert classification system (10), all 26 ACs (four cases of type I, 22 cases of type II) corresponded to 'carcinoma of the gastroesophageal junction'. Table I summarizes the clinicopathological findings of the patients. We
Table II. Antibodies used in this study.

\begin{tabular}{lccc}
\hline Antibody & & Source & Dilution \\
\hline p40 & Rabbit polyclonal & Nichirei Biosciences & Ready \\
p63 & 4A4 & Nichirei Biosciences & Ready \\
Cytokeratin 5/6 & D5/16 B4 & Dako & $1: 100$ \\
Cytokeratin & CAM5.2 & Becton, Dickinson & Ready \\
& \multicolumn{3}{|}{ and Company } \\
CDX2 & AMT28 & Leica & $1: 50$ \\
MUC-5AC & CLH2 & Leica & $1: 50$ \\
MUC-6 & CLH5 & Leica & $1: 50$ \\
\hline
\end{tabular}

used the WHO classification for histological diagnosis and grading when examining the HE-stained slides of all cases $(9,11)$. SCCs comprised three well-differentiated, nine moderately differentiated and three poorly differentiated tumors. The ACs included 11 cases of tubular adenocarcinoma, three poorly cohesive carcinomas and 12 mixed carcinomas. Of the 12 mixed carcinomas, the most common combination was tubular adenocarcinoma and poorly cohesive carcinoma in eight cases, followed by tubular and papillary adenocarcinoma in three cases, followed by poorly cohesive carcinoma, mucinous and tubular adenocarcinoma in one case. We diagnosed Barrett esophagus histopathologically in the 11 resected esophaguses from the $26 \mathrm{AC}$ cases. Two pathologists (K.M. and R.K.) independently reviewed HE stained slides blinded to the original pathological diagnosis. This study was approved by the Research Ethics Committee of Faculty of Medicine, University of Yamanashi.

Immunohistochemistry. Sections 4- $\mu$ m-thick were cut from formalinfixed, paraffin-embedded tissue blocks and then dewaxed and rehydrated. Immunohistochemical staining were performed on representative slides. Table II lists the primary antibodies used, their dilutions and their sources. Antigen retrieval was accomplished through various heat treatments before performing the primary antibody reactions: autoclaving at $120^{\circ} \mathrm{C}$ for $10 \mathrm{~min}$ in Antigen Retrieval Solution pH9 (Nichirei Biosciences, Tokyo, Japan) for p40, p63, cytokeratin 5/6, MUC-5AC and MUC-6; 0.1\% trypsin treatment at $37^{\circ} \mathrm{C}$ for $10 \mathrm{~min}$ in a water-bath for CAM5.2; and autoclaving at $120^{\circ} \mathrm{C}$ for $10 \mathrm{~min}$ in $1 \mathrm{mM}$ EDTA pH 8.0 for CDX2. After inhibiting endogenous peroxidase, we used positive controls to perform the primary antibody reactions. We used the N-Histofine Simple Stain MAX PO (MULTI) (Nichirei Biosciences) with diaminobenzidine as a chromogen and a light counterstain with hematoxylin to perform immunohistochemistry. Two pathologists (K.M. and R.K.) simultaneously reviewed immunostained sections using a double-headed light microscope. The extent of immunohistochemical staining was scored as $0(0 \%$ reactive tumor cells $), 1+(1 \%$ to $10 \%$ reactive tumor cells $), 2+(11 \%$ to $50 \%$ reactive tumor cells) or $3+(51 \%$ to $100 \%$ reactive tumor cells $)$. We defined $1+, 2+$ or $3+$ scored specimens as immunopositive and sections scored 0 as immunonegative.

Statistical analysis. We used the Fischer's exact test to evaluate differences between the SCC and AC samples of the EGJ regarding 
Table III. Immunohistochemical results of squamous cell carcinoma (SCC) and adenocarcinoma (AC) of the esophagogastric junction.

\begin{tabular}{lcccc}
\hline Markers & \multicolumn{4}{c}{ Immunohistochemical score* } \\
\cline { 2 - 5 } & 0 & $1+$ & $2+$ & $3+$ \\
\hline SCC (n=15) & & & & \\
p40 (\%) & $0(0 \%)$ & $0(0 \%)$ & $0(0 \%)$ & $15(100 \%)$ \\
p63 (\%) & $0(0 \%)$ & $0(0 \%)$ & $0(0 \%)$ & $15(100 \%)$ \\
Cytokeratin 5/6 (\%) & $0(0 \%)$ & $0(0 \%)$ & $0(0 \%)$ & $15(100 \%)$ \\
CAM5.2 (\%) & $4(27 \%)$ & $4(27 \%)$ & $2(13 \%)$ & $5(33 \%)$ \\
CDX2 (\%) & $15(100 \%)$ & $0(0 \%)$ & $0(0 \%)$ & $0(0 \%)$ \\
MUC-5AC (\%) & $13(87 \%)$ & $1(7 \%)$ & $1(7 \%)$ & $0(0 \%)$ \\
MUC-6 (\%) & $13(87 \%)$ & $2(13 \%)$ & $0(0 \%)$ & $0(0 \%)$ \\
AC (n=26) & & & & \\
p40 (\%) & $23(88 \%)$ & $2(8 \%)$ & $1(4 \%)$ & $0(0 \%)$ \\
p63 (\%) & $12(46 \%)$ & $10(38 \%)$ & $4(15 \%)$ & $0(0 \%)$ \\
Cytokeratin 5/6 (\%) & $21(81 \%)$ & $2(8 \%)$ & $3(12 \%)$ & $0(0 \%)$ \\
CAM5.2 (\%) & $0(0 \%)$ & $0(0 \%)$ & $0(0 \%)$ & $26(100 \%)$ \\
CDX2 (\%) & $5(19 \%)$ & $3(12 \%)$ & $7(27 \%)$ & $11(42 \%)$ \\
MUC-5AC (\%) & $6(23 \%)$ & $7(27 \%)$ & $9(35 \%)$ & $4(15 \%)$ \\
MUC-6 (\%) & $4(15 \%)$ & $10(38 \%)$ & $4(15 \%)$ & $8(31 \%)$ \\
\hline
\end{tabular}

CAM5.2: Anti-cytokeratin; CDX2: caudal-type homeobox 2; MUC: mucin. $*$ Score $0: 0 \%$ reactive tumor cells; $1+: 1 \%$ to $10 \%$ reactive tumor cells; $2+: 11 \%$ to $50 \%$ reactive tumor cells; and $3+: 51 \%$ to $100 \%$ reactive tumor cells.

immunohistochemical staining of p40, p63, cytokeratin 5/6, CAM5.2, CDX2, MUC-5AC and MUC-6. A p-value of less than 0.05 indicates statistical significance. Statistical analysis was carried out using the IBM SPSS Statistics version 22 (IBM Corp., Armonk, NY, USA) .

\section{Results}

Immunohistochemistry. Tables III, IV and V summarize the immunohistochemical results from the 15 SCCs and 26 ACs of the EGJ. Immunoreactivity for p40 of SCCs was $100 \%$ with a score of $3+$ (Figure 1B). Immunoreactivity for p63 of SCCs was $100 \%$ with a score of $3+$ (Figure 1D). Immunoreactivity for cytokeratin $5 / 6$ of SCCs was $100 \%$ with a score of $3+$ (Figure 1F). Immunoreactivity to CAM5.2 of SCCs (Figure 1H) varied. Immunoreactivity for CDX2 of SCCs was $100 \%$ with a score 0 (Figure $1 \mathrm{~J}$ ). Immunoreactivity for MUC-5AC of SCCs varied (Figure 1L).

Only two cases (13\%) of SCC showed any immunoreactivity for MUC-6 (Figure $1 \mathrm{~N}$ ) with a score of $1+$ staining. The sensitivities and specificities of p40, p63 and cytokeratin $5 / 6$ for the diagnosis of SCC were $100 \%$ and $88 \%, 100 \%$ and $46 \%$, and $100 \%$ and $81 \%$, respectively.

Immunoreactivity for $\mathrm{p} 40, \mathrm{p} 63$, cytokeratin $5 / 6, \mathrm{CDX} 2$, MUC-5AC, MUC-6 of ACs varied (Figure 2B, D, F, J, L, N). Immunoreactivity for CAM5.2 of ACs was $100 \%$ with a score of $3+$ (Figure $2 \mathrm{H}$ ). The sensitivities and specificities
Table IV. Sensitivities and specificities of markers of squamous cell carcinoma (SCC) and adenocarcinoma (AC) of the esophagogastric junction.

\begin{tabular}{|c|c|c|c|c|}
\hline \multirow[t]{2}{*}{ Marker } & \multicolumn{2}{|c|}{ Sensitivity* } & \multirow[t]{2}{*}{ Specificity* } & \multirow[t]{2}{*}{$p$-Value } \\
\hline & $\operatorname{SCC}(n=15)$ & $\mathrm{AC}(\mathrm{n}=26)$ & & \\
\hline \multicolumn{5}{|l|}{ SCC } \\
\hline $\mathrm{p} 40$ & $100 \%$ & $12 \%$ & $88 \%$ & $<0.001$ \\
\hline p63 & $100 \%$ & $54 \%$ & $46 \%$ & 0.001 \\
\hline Cytokeratin 5/6 & $100 \%$ & $19 \%$ & $81 \%$ & $<0.001$ \\
\hline \multicolumn{5}{|l|}{$\mathrm{AC}$} \\
\hline CAM5.2 & $73 \%$ & $100 \%$ & $27 \%$ & 0.013 \\
\hline $\mathrm{CDX} 2$ & $0 \%$ & $81 \%$ & $100 \%$ & $<0.001$ \\
\hline MUC-5AC & $13 \%$ & $77 \%$ & $87 \%$ & $<0.001$ \\
\hline MUC-6 & $13 \%$ & $85 \%$ & $87 \%$ & $<0.001$ \\
\hline
\end{tabular}

CAM5.2: Anti-cytokeratin; CDX2: caudal-type homeobox 2; MUC: mucin. *Data for any amount of reactivity considered positive for SCC vs. AC.

Table V. Immunohistochemical results for the combination of $\mathrm{p} 40 /$ caudal-type homeobox 2 (CDX2) in squamous cell carcinoma (SCC) and adenocarcinoma $(A C)$ of the esophagogastric junction.

\begin{tabular}{lccr}
\hline Marker status* & SCC $(\mathrm{n}=15)$ & AC $(\mathrm{n}=26)$ & $p$-Value \\
\hline $\mathrm{p} 40^{+} / \mathrm{CDX}^{-}$ & $15(100 \%)$ & $1(4 \%)$ & $<0.001$ \\
$\mathrm{p} 40^{-} / \mathrm{CDX2}^{+}$ & $0(0 \%)$ & $19(73 \%)$ & $<0.001$ \\
$\mathrm{p} 40^{+} / \mathrm{CDX}^{+}$ & $0(0 \%)$ & $2(8 \%)$ & 0.524 \\
$\mathrm{p} 40^{-} / \mathrm{CDX} 2^{-}$ & $0(0 \%)$ & $4(15 \%)$ & 0.278 \\
\hline
\end{tabular}

*Data for any amount of reactivity considered positive.

for CAM5.2, CDX2, MUC-5AC and MUC-6 of the diagnosis of $\mathrm{AC}$ were $100 \%$ and $27 \%, 81 \%$ and $100 \%, 77 \%$ and $87 \%$, and $85 \%$ and $87 \%$, respectively. However, the combination of $\mathrm{p} 40$ positivity and CDX2 negativity was seen in $100 \%$ of SCCs, with a specificity of $96 \%$, and the combination of $\mathrm{p} 40$ negativity and CDX2 positivity was seen in $73 \%$ of the ACs, with a specificity of $100 \%$.

Although immunoreactivities for p40, p63 or cytokeratin $5 / 6$ were almost the same in well-versus moderately versus poorly differentiated SCCs, CAM5.2 tended to immunostain more widely in moderate to poorly differentiated SCCs. However, we confirmed weak or lack of expression of p40 and p63 in keratinizing SCC cells. Although the better differentiated areas (carcinoma cells within glandular or papillary structures in particular) of the ACs tended to have CDX2, MUC-5AC and MUC-6 immunostaining, p40 or cytokeratin $5 / 6$ tended to stain in the poorly differentiated areas (carcinoma cells that were isolated or arranged in small aggregates in particular) of the ACs. 

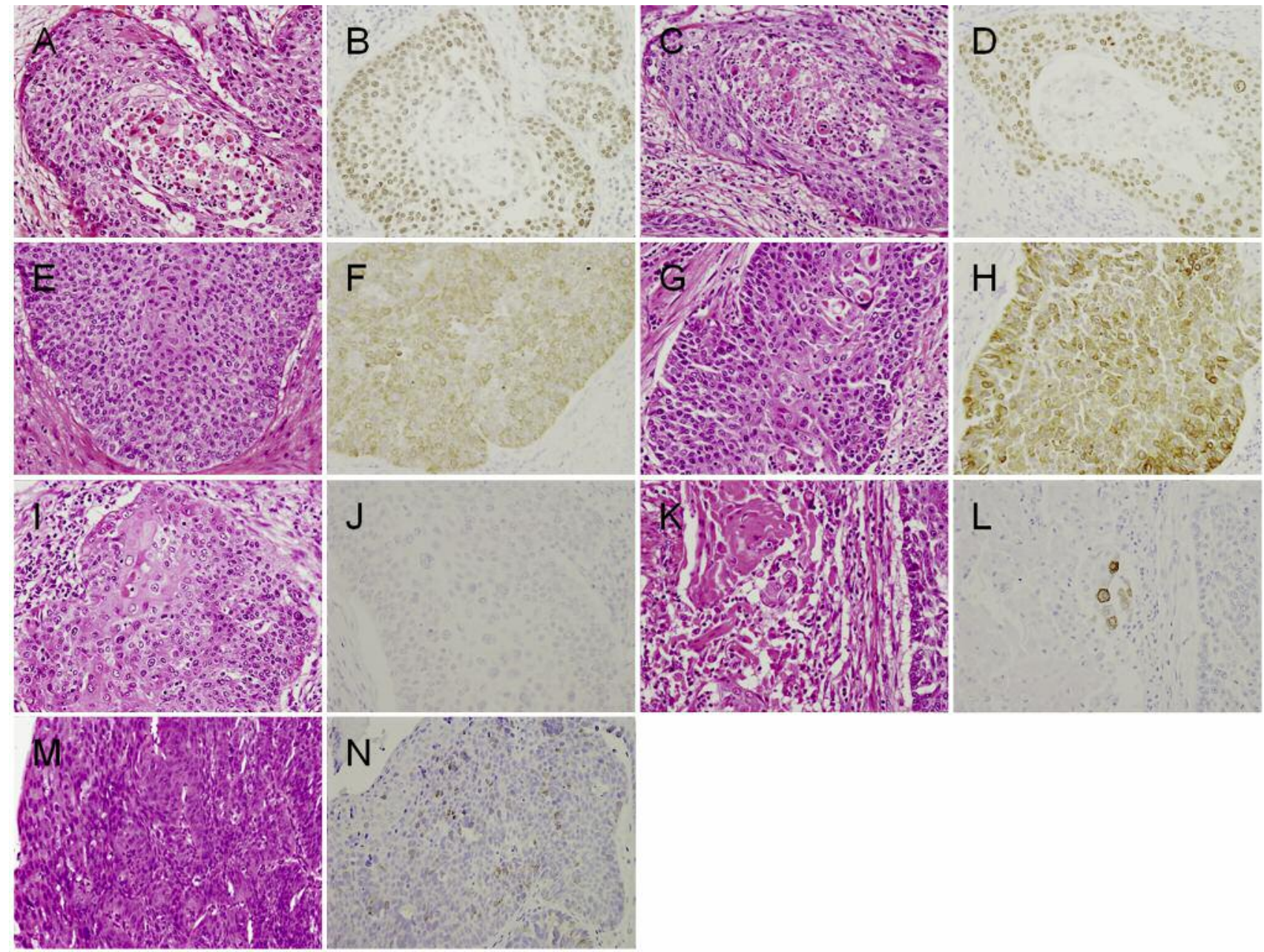

Figure 1. Representative images of tissue sections of squamous cell carcinomas (SCCs) of the esophagogastric junction as visualized with hematoxylin and eosin stain $(A, C, E, G, I, K$ and $M)$ and sections stained by immunohistochemistry: p40 (B) and p63 (D) immunoreactivity in nuclei; cytokeratin 5/6 (F) and CAM5.2 (H) immunoreactivity in the cytoplasm. SCCs generally exhibited no caudal-type homeobox 2 immunoreactivity $(J)$, and scant mucin $(M U C)-5 A C(L)$ and MUC-6 $(N)$ immunoreactivity in the cytoplasm exhibiting. Original magnification $\times 200$.

Statistical analysis. Using the two-tailed Fischer's exact test, SCC and AC cases of the EGJ statistically significantly differentially stained for $\mathrm{p} 40(p<0.001)$, p63 $(p=0.001)$, cytokeratin 5/6 $(p<0.001)$, CAM5.2 $(p=0.013)$, CDX2 $(p<0.001)$, MUC-5AC $(p<0.001)$ and MUC-6 $(p<0.001)$ individually, and using the combination of $\mathrm{p} 40$ and CDX2 $(p<0.001)$.

\section{Discussion}

The findings in this study confirm and expand upon several recent reports, suggesting that the p40 antibody is markedly superior to the standard p63 antibody in the diagnosis of SCC (3-5); however, there are few reports on the expression of p40 in esophageal SCCs $(7,12)$. Bishop et al. highlighted the important finding that p40 was equivalent to p63 in sensitivity for pulmonary SCC: all SCCs were positive for both markers, and reactivity for both markers was consistently diffuse (3). Furthermore, another important finding was that p40 was markedly superior to p63 in specificity: although p63 showed significant reactivity in pulmonary ACs ( $31 \%$ of cases), only rarely did ACs (3\% of cases) have labeling for $\mathrm{p} 40$, and when they did it was always focal (3). We found that the sensitivity of p40 for SCC of the EGJ was $100 \%$ and specificity was $88 \%$, while those for p63 were $100 \%$ and $46 \%$, respectively. These findings indicate that routine use of $\mathrm{p} 40$ in place of $\mathrm{p} 63$ would be more appropriate in order to differentiate SCC from $\mathrm{AC}$ of the EGJ similarly to the discrimination between pulmonary SCC and AC (3). 

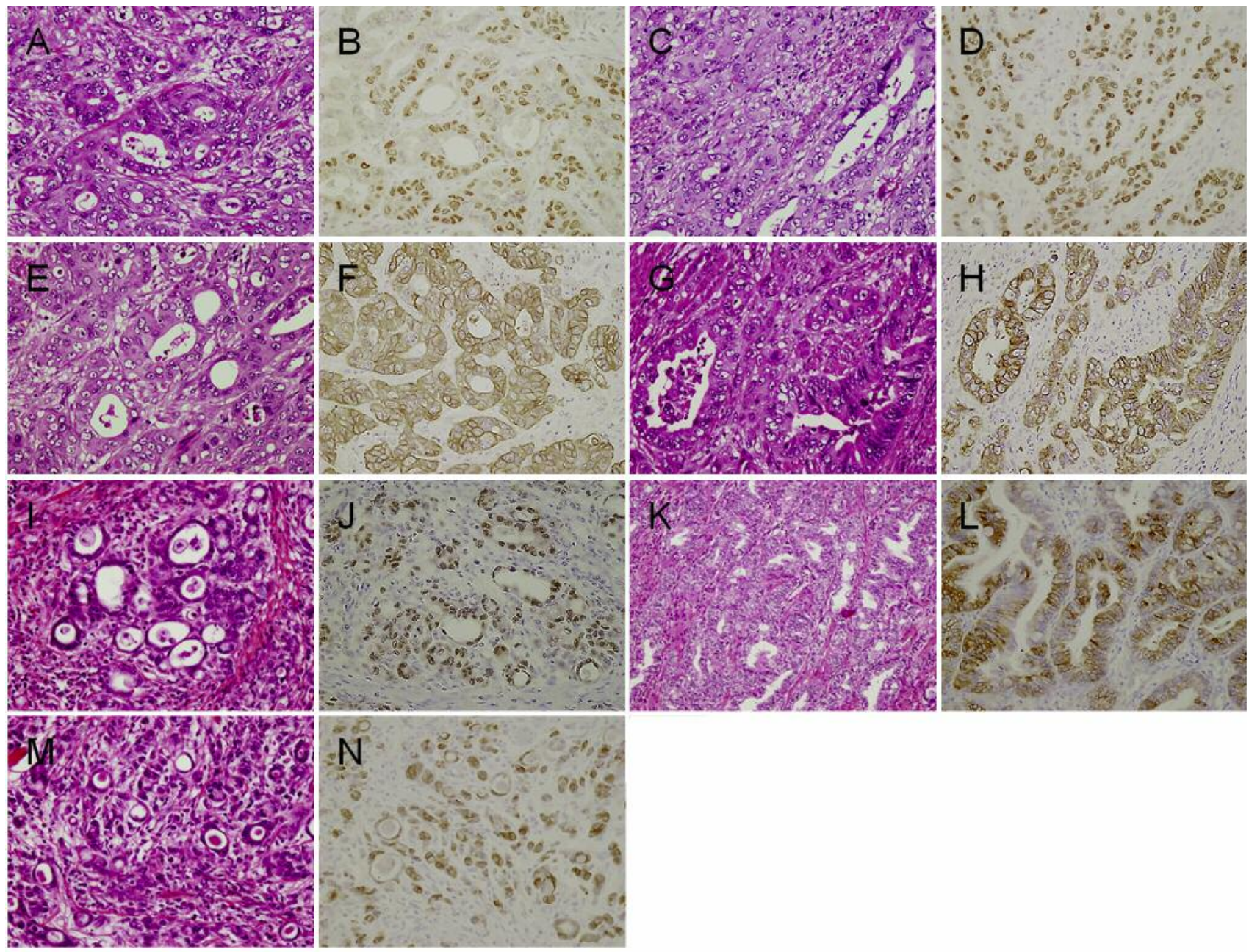

Figure 2. Representative images of tissue sections of adenocarcinomas (ACs) of the esophagogastric junction as visualized with hematoxylin and eosin stain ( $A, C, E, G, I, K$ and $M)$ and corresponding sections stained by immunohistochemistry: $p 40(C), p 63(D)$ and caudal-type homeobox 2 $(J)$ immunoreactivity in the nuclei; cytokeratin 5/6 (F) and CAM5.2 (H), mucin (MUC)-5AC (L) and MUC-6 (N) immunoreactivity in the cytoplasm. Original magnification $\times 200$.

The individual markers that were most sensitive and specific for diagnosing SCC of the EGJ were the presence of any 40 (sensitivity, $100 \%$; specificity, $88 \%$ ) or cytokeratin $5 / 6$ (sensitivity, $100 \%$; specificity, $81 \%$ ) staining. These findings indicate that $\mathrm{p} 40$ is slightly superior to cytokeratin 5/6 in specificity for diagnosing SCC of the EGJ, whereas CAM5.2 appears to be the most sensitive marker for AC of the EGJ but suffers from the lowest specificity (sensitivity, 100\%; specificity, 27\%). In contrast, CDX2 is highly sensitive and the most specific marker for AC of the EGJ (sensitivity, 81\%; specificity, 100\%).

The combination of 440 positivity and CDX2 negativity was seen in $100 \%$ of the 15 EGJ SCCs with a specificity of $96 \%(p<0.001)$, whereas the combination of $\mathrm{p} 40$ negativity and CDX2 positivity was seen in $73 \%$ of the 26 EGJ ACs with a specificity of $100 \%(p<0.001)$. Moreover, immunoreactivity for p40 was consistently diffuse (immunohistochemical score $3+)$ in even poorly differentiated SCCs. This two marker panel is highly sensitive and specific for distinguishing SCCs from ACs of the EGJ. Recently, we encountered a biopsy specimen of Borrmann type 2 EGJ carcinoma (male; 67 years old). Because it lacked specific architectural or cytological features by histomorphologic examination of a HE-stained slide under a microscope (Figure 3A and B), we could not determine the accurate histological subclassification. Consequently, we applied our study result and were able to diagnose it as poorly differentiated adenocarcinoma due to p40 immunonegativity and CDX2 immunopositivity (Figure 3C and D).

Demicco et al. defined an immunophenotype of ACs of the distal esophagus or EGJ. They classified the tumor as 


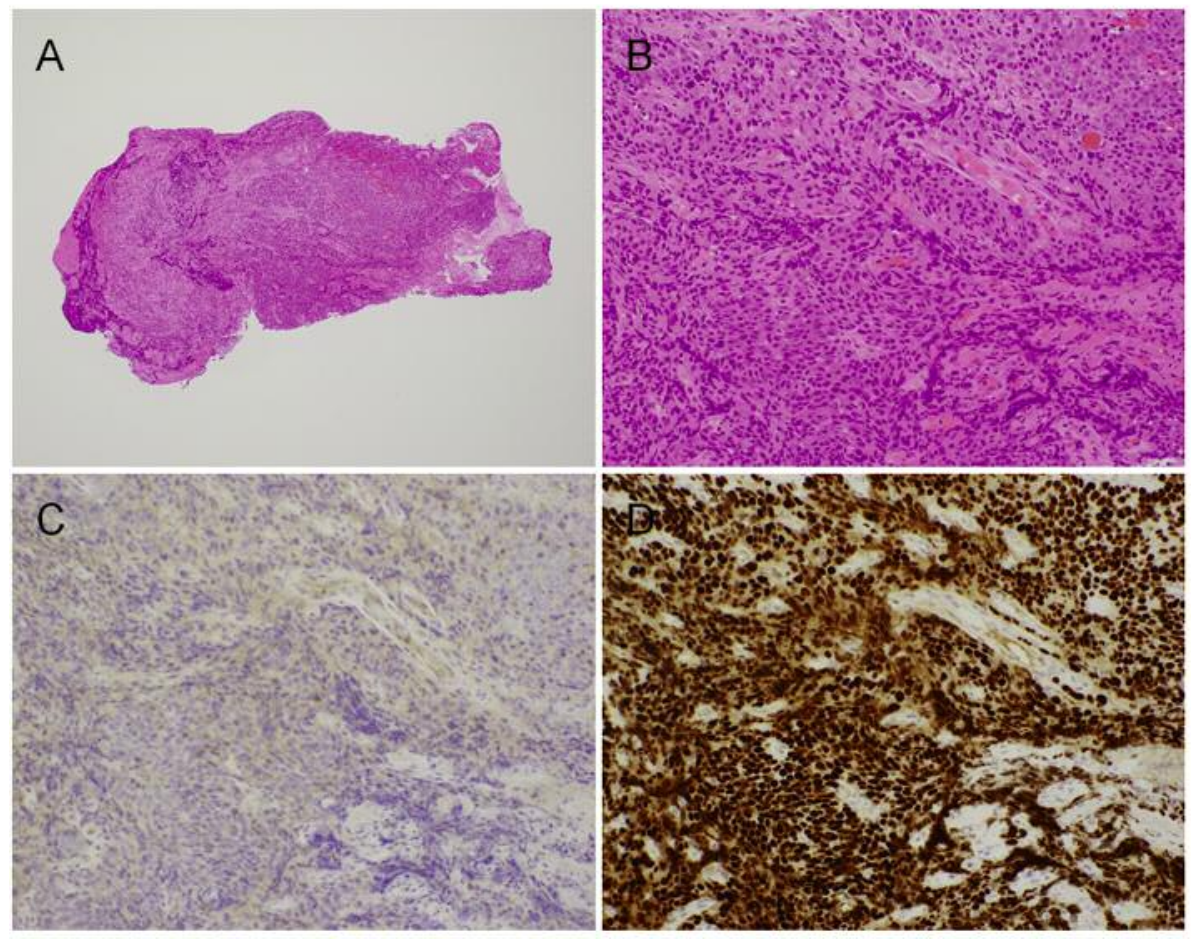

Figure 3. The biopsy specimen of Borrmann type 2 esophagogastric junction carcinoma. A, B: Hematoxylin and eosin stain (original magnification $\times 40, \times 200$, respectively). Carcinoma lacked characteristic architecture and had crush artifact in the specimen preparation. C: The carcinoma exhibited no p40 immunoreactivity (original magnification $\times 200$ ). D: The carcinoma exhibited caudal-type homeobox 2 immunoreactivity in the nuclei (original magnification $\times 200$ ).

intestinal if it stained positively for CDX2, CD10 or MUC2, and also stained negatively for both MUC-5AC and MUC6. They classified the tumor as gastric if it was positive for MUC-5AC or MUC-6 and also negative for CDX2, CD10 and MUC-2. They classified the tumor as mixed if it was positive for any combination of both intestinal and gastric markers, or null if all the core samples from a case were negative for all five markers (13). They reported that 38 out of 157 cases $(24 \%)$ of ACs of the distal esophagus or EGJ were intestinal, 41 cases $(26 \%)$ were gastric, 37 cases $(24 \%)$ were mixed and 21 cases (13\%) were null type (13). Based on their analysis, if both p40 and CDX2 immunoreactivity is negative in the tumor cells, additional studies with MUC5AC and MUC-6 may help confirm the diagnosis of gastric type AC of the EGJ.

In conclusion, we demonstrated that $\mathrm{p} 40$ is equivalent to p63 in sensitivity for SCC of the EGJ, but it had a distinct advantage over p63 in its remarkably strong specificity. While we found that the two-marker panel of p40 and CDX2 is highly sensitive and specific for distinguishing SCC from AC of the EGJ. Moreover, immunoreactivity for p40 was consistently diffuse (immunohistochemical score $3+$ ) in even poorly differentiated SCCs. The combination of these two biomarkers can help differentiate SCC from AC of the EGJ, especially when only small biopsy specimens are available. Furthermore, the presence of MUC-5AC or MUC-6 immunoreactivity may help diagnose a gastric-type $\mathrm{AC}$ tumor of the EGJ when both p40 and CDX2 are negative.

\section{Conflicts of Interest}

None of the Authors have any conflict of interest in regard to this study.

\section{References}

1 Bang YJ, Van Cutsem E, Feyereislova A, Chung HC, Shen L, Sawaki A, Lordick F, Ohtsu A, Omuro Y, Satoh T, Aprile G, Kulikov E, Hill J, Lehle M, Rüschoff $\mathrm{J}$ and Kang YK: Trastuzumab in combination with chemotherapy versus chemotherapy alone for treatment of HER2-positive advanced gastric or gastro-oesophageal junction cancer (ToGA): a phase 3, open-label, randomised controlled trial. Lancet 376: 687-697, 2010.

2 Zhang $\mathrm{K}$, Deng $\mathrm{H}$ and Cagle PT: Utility of immunohistochemistry in the diagnosis of pleuropulmonary and mediastinal cancers: a review and update. Arch Pathol Lab Med 138: 1611-1628, 2014. 
3 Bishop JA, Teruya-Feldstein J, Westra WH, Pelosi G, Travis WD and Rekhtman N: p40 ( $\Delta \mathrm{Np} 63)$ is superior to $\mathrm{p} 63$ for the diagnosis of pulmonary squamous cell carcinoma. Mod Pathol 25: 405-415, 2012.

4 Bir F, Aksoy Altınboga A, Satiroglu Tufan NL, Kaya S, Baser S and Yaren A: Potential utility of p63 expression in differential diagnosis of non-small-cell lung carcinoma and its effect on prognosis of the disease. Med Sci Monit 20: 219-226, 2014.

5 Collins BT, Wang JF and Bernadt CT: Utilization of p40 $(\Delta \mathrm{Np} 63)$ with p63 and cytokeratin $5 / 6$ immunohistochemistry in non-small cell lung carcinoma fine-needle aspiration biopsy. Acta Cytol 57: 619-624, 2013.

6 Geddert H, Kiel S, Heep HJ, Gabbert HE and Sarbia M: The role of p63 and deltaNp63 (p40) protein expression and gene amplification in esophageal carcinogenesis. Hum Pathol 34: 850856,2003

7 Long KB and Hornick JL: SOX2 is highly expressed in squamous cell carcinomas of the gastrointestinal tract. Hum Pathol 40: 1768-1773, 2009.

8 DiMaio MA, Kwok S, Montgomery KD, Lowe AW and Pai RK: Immunohistochemical panel for distinguishing esophageal adenocarcinoma from squamous cell carcinoma: a combination of p63, cytokeratin 5/6, MUC5AC, and anterior gradient homolog 2 allows optimal subtyping. Hum Pathol 43: 17991807, 2012.

9 Odze RD, Fléjou JF, Boffetta P, Höfler H, Montgomery E and Spechler SJ: Adenocarcinoma of the oesophagogastric junction. In: WHO Classification of Tumors of the Digestive System. Bosman FT, Carneiro F, Hruban RH and Theise ND (eds.). IARC press, Lyon, pp. 40-44, 2010.
10 Siewert JR and Stein HJ: Carcinoma of the cardia: Carcinoma of the gastroesophageal junction - classification, pathology and extent of resection. Dis Esophagus 9: 173-182, 1996.

11 Montgomery E, Fielf JK, Boffetta P, Daigo Y, Shimizu M and Shimoda T: Squamous cell carcinoma of the esophagus. In: WHO Classification of Tumors of the Digestive System. Bosman FT, Carneiro F, Hruban RH and Theise ND (eds.). IARC press, Lyon, pp. 18-24, 2010.

12 Morita M, Uramoto H, Nakata S, Ono K, Sugaya M, Yoshimatsu T, Oyama T, Hanagiri T, Sugio K and Yasumoto K: Expression of deltaNp63 in squamous cell carcinoma of the esophagus. Anticancer Res 25: 3533-3539, 2005.

13 Demicco EG, Farris AB 3rd, Baba Y, Agbor-Etang B, Bergethon K, Mandal R, Daives D, Fukuoka J, Shimizu M, Dias-Santagata D, Ogino S, Iafrate AJ, Gaissert HA and Mino-Kenudson M: The dichotomy in carcinogenesis of the distal esophagus and esophagogastric junction: intestinal-type vs. cardiac-type mucosa-associated adenocarcinoma. Mod Pathol 24: 1177-1190, 2011.
Received September 5, 2017

Revised September 24, 2017

Accepted September 27, 2017 